\title{
Prevalence of Human Papillomavirus, Chlamydia trachomatis, and Neisseria gonorrhoeae in Commercial Sex Workers in Japan
}

\author{
Kazuhisa Ishi, ${ }^{1 *}$ Fujihiko Suzuki, ${ }^{1}$ Akira Saito, ${ }^{1}$ and \\ Takeyoshi Kubota ${ }^{2}$ \\ ${ }^{1}$ Department of Clinical Pathology, Juntendo University, Urayasu Hospital, Chiba, Japan \\ ${ }^{2}$ Department of Obstetrics and Gynecology, Juntendo University, Urayasu Hospital, Chiba, Japan
}

\begin{abstract}
Objective: We used the hybrid capture assays to investigate the prevalence of human papillomavirus (HPV), Chlamydia trachomatis and Neisseria gonorrhoeae among commercial sex workers in Tokyo.

Methods: Five hundred forty-six consecutive commercial sex workers (CSW) who visited an STD clinic for STD checkup in 1998 and 1999 were studied. A control group consisted of 233 consecutive women who visited a general gynecological clinic for annual checkup. A cervical sample was obtained for hybrid capture assays for HPV-A (low-oncogenic-risk types), HPV-B (intermediate- and high-oncogenic-risk types), C. trachomatis, and N. gonorrhoeae. Fisher's exact test was used for statistical analyses.

Results: The positive rate for HPV-B among the CSW was 48.4\%, significantly higher than the $6.0 \%$ among the control subjects. The positive rates for HPV-A, C. trachomatis, and N. gonorrhoeae were also significantly higher among the CSW than among the control subjects. Among the microorganisms tested, the positive rate for HPV-B was the highest in both the STD and control groups.

Conclusions: The high prevalence of HPV, C. trachomatis, and N. gonorrhoeae infection in CSW poses a risk of further transmission of STD to the general public, suggesting the need for further education and screening for CSW and the general public. We found high- and intermediateoncogenic-type HPV to be the most prevalent infection among both CSW and control subjects. Screening for HPV may be necessary in STD and general clinics to predict the risk of cervical malignancy. Hybrid capture assays, which permit simultaneous detection of HPV and other STD with high sensitivity, may be a useful diagnostic method. Infect. Dis. Obstet. Gynecol. 8:235-239, 2000. () 2000 Wiley-Liss, Inc.
\end{abstract}

KEY WORDS

human papillomavirus; HPV; Chlamydia trachomatis; Neisseria gonorrhoeae; commercial sex workers; STD

Sinting exually transmitted diseases (STD) are all infections transmitted through sexual activities and consist of a diversity of diseases. Nongonococcal urethritis in both males and females and cervicitis are the most prevalent STD in Japan, most cases of which are caused by Chlamydia trachomatis. The second most prevalent STD have been reported to be Neisseria gonorrhoeae infection in males and herpes simplex infection in females. ${ }^{1,2}$ In addition, infection by human papillomavirus (HPV),

*Correspondence to: Kazuhisa Ishi, MD, Department of Clinical Pathology, Juntendo University Urayasu Hospital, 2-1-1 Tomioka, Urayasu City, Chiba 279-0021, Japan. E-mail: ishi3988@kt.rim.or.jp 
which has been implicated to be associated with cervical cancer, is increasingly important clinical problem. ${ }^{3}$ A simple and reliable method to detect HPV infection has not been available until now. Therefore, diagnostic and epidemiological studies on HPV have not been conducted adequately in Japan. Furthermore, commercial sex workers (CSW) have been reported to be an important source of STD infection, which implies a high possibility of STD transmission to ordinary families. ${ }^{2}$ Therefore, for the purpose of accurate diagnosis and treatment, a simple, rapid, and reliable diagnostic method for STD is necessary. Currently, culture methods and enzyme-labeled antibody reactions remain the main clinical diagnostic methods for $C$. trachomatis and $N$. gonorrhoeae identification, although new DNA detection techniques using highly specific DNA probes and gene amplification techniques, such as polymerase chain reaction and ligase chain reaction, have been developed and are gaining popularity. ${ }^{2}$ In this study, we used the hybrid capture method, ${ }^{4-7}$ a new DNA diagnostic method using a novel signal amplification technique, to investigate the prevalence of HPV, $C$. trachomatis, and $N$. gonorrhoeae among CSW who attended a STD clinic in an entertainment area in Tokyo.

\section{SUBJECTS AND METHODS}

We enrolled 546 consecutive CSW who visited an STD clinic in a metropolitan entertainment area for STD checkup between June, 1998, and September, 1999, in the study. Their ages ranged from 18 to 48 years, with a mean of $28.9 \pm 5.1$ years (mean \pm SD). A control group consisted of 233 consecutive women who visited the Department of Obstetrics and Gynecology, Juntendo Urayasu Hospital, Juntendo University, for annual routine checkup between October, 1998, and September, 1999. Their ages ranged from 17 to 45 years, with a mean of $31.0 \pm 6.5$ years.

A sample for hybrid capture assays was obtained by firmly wiping a swab with a Dacron stick across the cervix and external os. The swab was immediately placed into a specimen collection tube containing $1.0 \mathrm{ml}$ of preserving fluid and stored at $-80^{\circ} \mathrm{C}$ until it was assayed.

Hybrid capture assays for HPV, C. trachomatis, and $N$. gonorrhoeae were performed using Hybrid
Capture Assay Kits (Digene Diagnostics). ${ }^{4-7}$ The kit for HPV contained two separate HPV probe mixtures. One probe mixture, designated $A$, detects low-oncogenic-risk HPV types, including types $6,11,42,43$, and 44 . The other probe mixture, designated $\mathbf{B}$, detects high- and intermediateoncogenic-risk HPV types, including types 16, 18, $31,33,35,39,45,51,52,56,58,59$, and 68 . The kits for $G$. trachomatis and $N$. gonorrhoeae contained the respective probes for the two microorganisms. The assays were performed according to the directions of the manufacture as explained in previous reports. ${ }^{5}$ Briefly, the specimen was denatured, and a ribonucleic acid (RNA) probe was added for hybridization. The DNA-RNA hybrids were captured on the surface of a microplate coated with antiDNA-RNA hybrid antibodies and then reacted with another anti-DNA-RNA hybrid antibody mixture conjugated with alkaline phosphatase. The RNA-DNA hybrids were detected by chemoluminescence. Samples were considered positive when the index value (test sample value/positive control value) was $\geq 1.0$, and negative if the index value was $<1.0$. Fisher's exact test was used for statistical analyses. A $P$ value $<0.05$ was considered significant.

\section{RESULTS}

Table 1 shows the results of the hybrid capture assays. Among $546 \mathrm{CSW}, 218$ women were negative for all four probes [low-risk HPV types (HPVA), high- and intermediate-risk HPV types (HPVB), C. trachomatis, and N. gonorrhoeae]. One hundred eighty-four women were positive for HPV-B alone, 35 for $G$. trachomatis and HPV-B, 30 for both HPV-A and -B, 30 for HPV-A alone, 19 for $C$. trachomatis alone, 7 for $N$. gonorrhoeae alone, and 6 for HPV-A and -B and $C$. trachomatis. The other patterns had fewer than four women each. Among 233 control women, 211 were negative for all four probes. Nine women were positive for HPV-B alone. The other patterns consisted of fewer than four subjects each.

Table 2 compares the positive rates for HPV, $C$. trachromatis, and $N$. gonorrhoeae between CSW and control subjects. The positive rate for HPV-B among the CSW was $48.4 \%$ (264 of 546), significantly higher than the $6.0 \%$ (14 of 233) in the control group $(P<0.01)$. The positive rates for the 
TABLE I. Results of hybrid capture assays for HPV, C. trachomatis, and N. gonorrhoeae in the uterine cervix in commercial sex workers'

\begin{tabular}{|c|c|c|c|c|c|c|}
\hline & \multicolumn{4}{|c|}{ Hybrid capture assays } & \multirow{2}{*}{$\begin{array}{c}\text { Number of } \\
\text { CSW }\end{array}$} & \multirow{2}{*}{$\begin{array}{c}\text { Number of } \\
\text { normal controls }\end{array}$} \\
\hline & HPV A & HPV B & C. trachomatis & N. gonorrhoeae & & \\
\hline \multicolumn{7}{|l|}{ All tests } \\
\hline negative & - & - & - & - & 218 & 211 \\
\hline One test & + & - & - & - & 30 & 3 \\
\hline \multirow[t]{3}{*}{ positive } & - & + & - & - & 184 & 9 \\
\hline & - & - & + & - & 19 & 4 \\
\hline & - & - & - & + & 7 & 0 \\
\hline Two tests & + & + & - & - & 30 & 3 \\
\hline \multirow[t]{5}{*}{ positive } & + & - & + & - & 2 & 0 \\
\hline & + & - & - & + & 2 & 0 \\
\hline & - & + & + & - & 35 & 2 \\
\hline & - & + & - & + & 2 & 0 \\
\hline & - & - & + & + & 3 & 1 \\
\hline Three tests & + & + & + & - & 6 & 0 \\
\hline \multirow{3}{*}{ positive } & + & + & - & + & 2 & 0 \\
\hline & + & - & + & + & 1 & 0 \\
\hline & - & + & + & + & 3 & 0 \\
\hline $\begin{array}{l}\text { Four tests } \\
\text { positive }\end{array}$ & + & + & + & + & 2 & 0 \\
\hline Total & & & & & 546 & 233 \\
\hline
\end{tabular}

'HPV-A, low-oncogenic-risk HPV types; HPV-B, high- and intermediate-oncogenic-risk HPV types; CSW, commercial sex workers.

TABLE 2. Comparison of positive rates for HPV, C trachomatis, and N. gonorrhoeae between CSW and control women: positive results of hybrid capture assays performed on cervical samples'

\begin{tabular}{|c|c|c|c|c|c|c|c|c|c|}
\hline & \multicolumn{2}{|c|}{ HPV-A } & \multicolumn{2}{|c|}{ HPV-B } & \multicolumn{2}{|c|}{ C. trachomatis } & \multicolumn{2}{|c|}{ N. gonorrhoeae } & \multirow[b]{2}{*}{ Total cases } \\
\hline & $\begin{array}{c}\text { Positive } \\
\text { cases }\end{array}$ & $\begin{array}{l}\text { Positive } \\
\text { rate (\%) }\end{array}$ & $\begin{array}{c}\text { Positive } \\
\text { cases }\end{array}$ & $\begin{array}{l}\text { Positive } \\
\text { rate (\%) }\end{array}$ & $\begin{array}{c}\text { Positive } \\
\text { cases }\end{array}$ & $\begin{array}{l}\text { Positive } \\
\text { rate (\%) }\end{array}$ & $\begin{array}{c}\text { Positive } \\
\text { cases }\end{array}$ & $\begin{array}{l}\text { Positive } \\
\text { rate (\%) }\end{array}$ & \\
\hline CSW & 75 & 13.7 & 264 & 48.4 & 71 & 13 & 22 & 4.1 & 546 \\
\hline Control group & 6 & 2.6 & 14 & 6 & 7 & 3 & I & 0.4 & 233 \\
\hline$P$ value & \multicolumn{2}{|c|}{$<0.01$} & \multicolumn{2}{|c|}{$<0.01$} & \multicolumn{2}{|c|}{$<0.05(P=0.011)$} & \multicolumn{2}{|c|}{$<0.01$} & \\
\hline
\end{tabular}

'HPV-A, low-oncogenic-risk HPV types; HPV-B: High- and intermediate-oncogenic-risk HPV types; CSW, commercial sex workers.

other three probes were also significantly higher among CSW than among the control subjects.

For the microorganisms tested, the positive rate for HPV-B was the highest in both the CSW and the control groups. There were no differences in the positive rate for HPV-B among different age groups in the CSW.

\section{DISCUSSION}

In Japan, owing to the flourishing sex industry and active sexuality among the young age group, STD, especially the largely asymptomatic but also symptomatic chlamydial and viral infections, have infiltrated the general society with considerable force. $^{1,2}$ The reported incidence of $N$. gonorrhoeae and $C$. trachomatis infections among CSW in Japan varies depending on investigators and the diagnos- tic methods used. The incidence of $N$. gonorrhoeae has been reported to be $3.8 \%,{ }^{8} 5.9 \%,{ }^{9}$ and $13.3 \%{ }^{10}$ and that of $C$. trachomatis to be $9.8 \%,{ }^{8} 15.1 \%,{ }^{9}$ and $17.4 \% .{ }^{10}$ However, the incidence of HPV in Japan, especially that among CSW, has not been reported. This is probably a result of the lack of a simple and effective detection method that can be used in clinical diagnosis or epidemiological studies.

In other countries, high detection rates of HPV among patients visiting STD clinics have been reported. Horn et al. ${ }^{11}$ and Figueroa et al. ${ }^{12}$ reported detection rates of $12 \%$ and $28.7 \%$, respectively, using Southern blot hybridization analysis, and Borg et al. ${ }^{13}$ reported $12 \%$ using dot blot hybridization analysis. Compared to these reported rates, our detection rate of HPV in CSW was higher (48.4\%). 
The reason is that the hybrid capture assay used in this study has higher sensitivity and specificity than Southern or dot blot hybridization methods, and our probes detect more HPV types than the previous studies. In the present survey, high- and intermediate-oncogenic-risk HPV were detected in nearly half of the CSW studied, and the positive rate in CSW was extremely high compared to the control subjects. Furthermore, the detection rate of high- and intermediate-oncogenic-type HPV was also higher than those of $N$. gonorrhoeae and $C$. trachomatis in control subjects. Therefore, we may assume that HPV is the most prevalent STD in Japan. HPV infection has attracted attention recently as a cause of cervical cancer. Diagnosis of HPV infection is important for the prediction of the risk of cervical intraepithelial neoplasia and cervical cancer.

We detected $C$. trachomatis and $N$. gonorrhoeae DNA in $13.0 \%$ and $4.1 \%$, respectively, of the samples from CSW. These figures are significantly higher than the positive rates found in our control subjects (3\% and $0.5 \%$, respectively) and those reported for the general Japanese female population. Positive rates of $0 \%$ for $N$. gonorrhoea ${ }^{2,9}$ and about $10 \%$ for $G$. trachomatis $^{2,14}$ have been reported for general married pregnant women. Compared to these figures, the positive rates among CSW are markedly elevated. The proportions of male STD cases having a source of infection from CSW vary depending on patients' age group. Figures such as approximately $70 \%$ of gonococcal urethritis and $40 \%$ of chlamydial urethritis in the 25 years or older age group, ${ }^{9} 64.3 \%$ of gonococcal urethritis and $54.3 \%$ of chlamydial urethritis, ${ }^{10}$ and $85 \%$ of all STD in males ${ }^{15}$ have been reported. Although the figures vary in different facilities, the carrier rate of STD in CSW is strongly associated with the incidence of STD in males. ${ }^{2}$

Hybrid capture method is a new, highly sensitive DNA diagnostic method using a novel signal amplification technique that does not require DNA amplification. In this method, long-chain RNA probes are used, and the DNA-RNA hybrids formed are detected immunologically using specific antibodies. Because one molecule of DNA binds with many antibodies, signals are amplified, achieving a high sensitivity of detection. The method is essentially an immunoassay, so there is no need for special equipment, and the procedures are simple. Hybrid capture method has been reported to have almost the same sensitivity and specificity as PCR for detecting HPV as the culture method and PCR for detecting $N$. gonorrhoeae. For detecting $C$. trachomatis, the hybrid capture method has the same sensitivity and specificity as PCR but is more sensitive than the culture method. ${ }^{5-7}$

HPV infection occurs frequently in conjunction with chlamydial and gonococcal infections. In the present study, these three microorganisms could easily be investigated simultaneously from one cervical swab sample using hybrid capture assays. By using these assays, the positive rates for HPV-A, HPV-B, C. trachomatis, and N. gonorrhoeae were significantly higher among the CSW than among the control subjects. For the four types of microorganisms, the positive rate for HPV-B was the highest. This was more prominent among the CSW than among the control subjects. Ichinose ${ }^{14}$ reported that the positive rates for $G$. trachomatis and $N$. gonorrhoeae among Japanese patients who visited general gynecologic clinics in Tokyo with symptoms of STD were $10.4 \%$ (1987-1997, $\mathrm{n}=50,535$, by either enzyme immunoassay or DNA probe method) and $3.7 \%(1992-1997, \mathrm{n}=5,872$, by $\mathrm{DNA}$ probe method), respectively. Compared to these results, the positive rates in our control subjects $(3.0 \%$ for $C$. trachomatis and $0.4 \%$ for $N$. gonorrhoeae) were lower, and the positive rates in the CSW $(13.0 \%$ for $C$. trachomatis and $4.1 \%$ for $N$. gonorrhoeae) were higher. Therefore, the present results are reasonable.

In Japan, restriction of oral contraceptive use was lifted in September, 1999, although use is still not widespread. There is much concern over the possible increase in STD transmission after the pills become widely available. Early control measures are required. STD checkup for CSW alone is inadequate; health education on the prevention of STD and screening for STD for the general public are also required. The present findings suggest that HPV examination is desirable for women who visit STD clinics to assess the future risk of cervical intraepithelial neoplasia and cervical cancer.

\section{REFERENCES}

1. Matsumoto T. Sexually transmitted diseases (STD). Rinsho Kensa (Clinical Laboratory Investigation) 1998; 42:1417-1427 (in Japanese). 
2. Kumamoto E, Tsukamoto Y, Nishitani I. Sexually transmitted diseases (STD)-chlamydia and various viruses. Saishin Igaku (Advances in Medicine) 1999;54:196-211 (in Japanese).

3. Wallin KL, Wiklund F, Angstrom T, et al. Typespecific persistence of human papillomavirus DNA before the development of invasive cervical cancer. $\mathrm{N}$ Engl J Med 1999;341:1633-1638.

4. Sun XW, Fererczy A, Johnson D. Evaluation of the hybrid capture human papillomavirus deoxyribonucleic acid detection test. Am J Obstet Gynecol 1995;173: 1432-1437.

5. Kubota T, Ishi K, Suzuki M, Utsuno S, Igari J. Usefulness of hybrid capture HPV DNA assay as a diagnostic tool for human papillomavirus infection. J Jpn Acad Infect Dis 1998;72:1219-1224.

6. Schachter J, Hook EW III, McCormack WM, et al. Ability of the digene hybrid capture II test to identify Chlamydia trachomatis and Neisseria gonorrhoeae in cervical specimens. J Clin Microbiol 1999;37:3668-3671.

7. Girdner JL, Cullen AP, Salama TG, He L, Lorincz A, Quinn TC. Evaluation of the digene hybrid capture II CT-ID test for detection of Chlamydia trachomatis in endocervical specimens. J Clin Microbiol 1999;37:1579_ 1581.

8. Hirose T, Nishimura M, Yoshio H. STD infection in commercial sex workers. Nihon Seikansen Shi (Japa- nese Journal of Sexually Transmitted Diseases) 1993;4: 13-22 (in Japanese).

9. Kashiwagi S. Status of HIV and STD infections in commercial sex worker. Report of the HIV Epidemiological Research Group of Medical and Welfare Scientific Research, 1996, Tokyo (in Japanese).

10. Tanaka M. Status of STD in Fukuoka City. Nihon Kansensho Shi (Japanese Journal of Infectious Diseases) 1993;4:39-46 (in Japanese).

11. Horn JE, McQuillan GM, Shan KV, et al. Genital human papillomavirus infections in patients attending an inner-city STD clinic. Sex Transm Dis 1991;18:183187.

12. Figueroa JP, Ward E, Luthi TE, et al. Prevalence of human papillomavirus among STD clinic attenders in Jamaica: association of younger age and increased sexual activity. Sex Transm Dis 1995;22:114-118.

13. Borg AJ, Medley G, Garland SM. Prevalence of HPV in a Melbourne female STD population: comparison of RNA and DNA probes in detecting HPV by dot blot hybridization. Int J STD AIDS 1993;4:159-164.

14. Ichinose M. Epidemiological study of Chlamydia trachomatis infection. Nihon Kansensho Shi (Japanese Journal of Infectious Diseases) 1996;7:24-31 (in Japanese).

15. Oosato H. Status of STD in Osaka City. Nihon Kansensho Shi (Japanese Journal of Infectious Diseases) 1993;4:34-38 (in Japanese). 


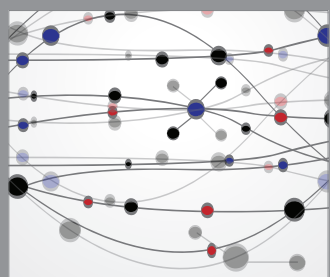

The Scientific World Journal
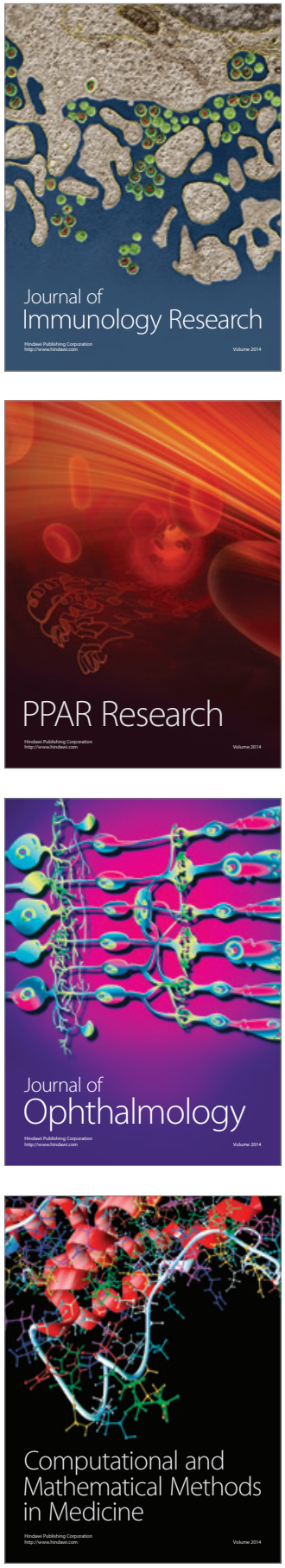

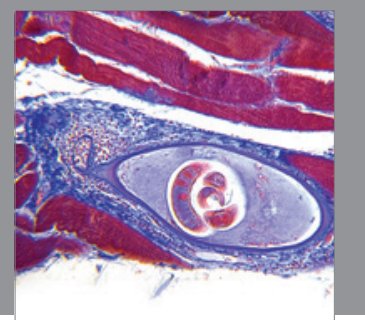

Gastroenterology

Research and Practice
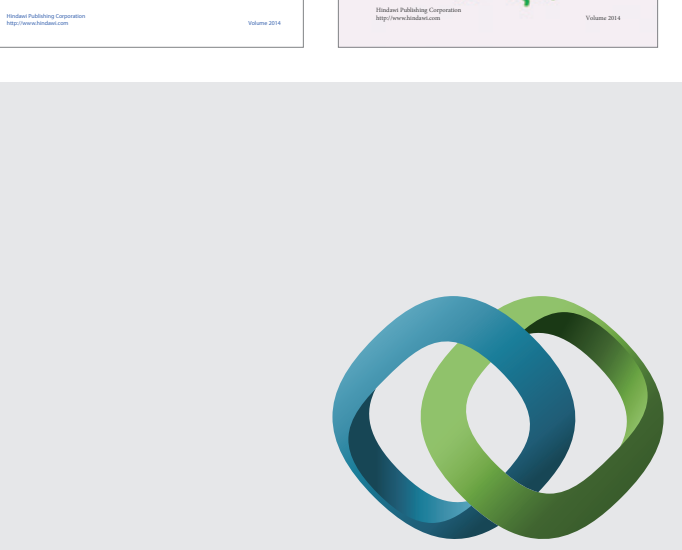

\section{Hindawi}

Submit your manuscripts at

http://www.hindawi.com
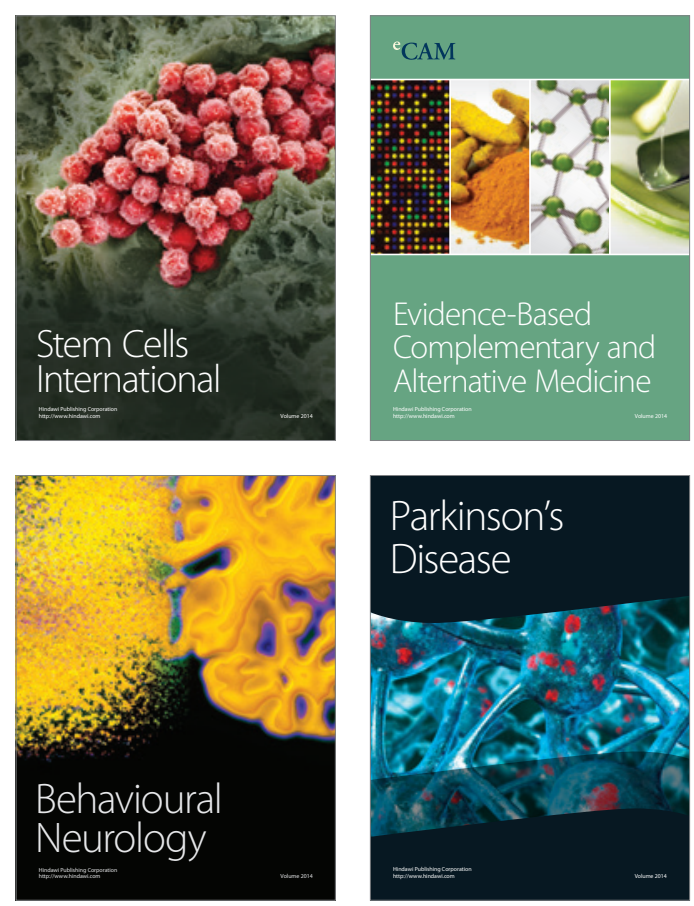

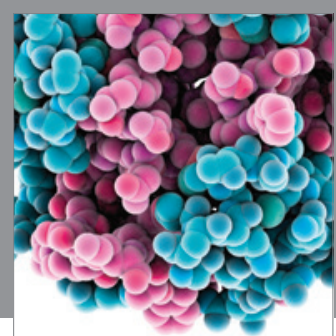

Journal of
Diabetes Research

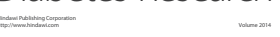

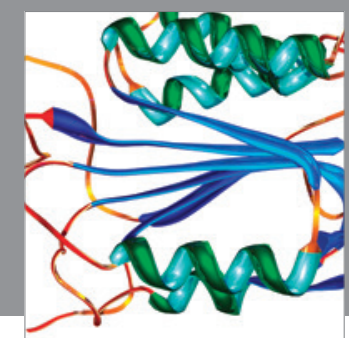

Disease Markers
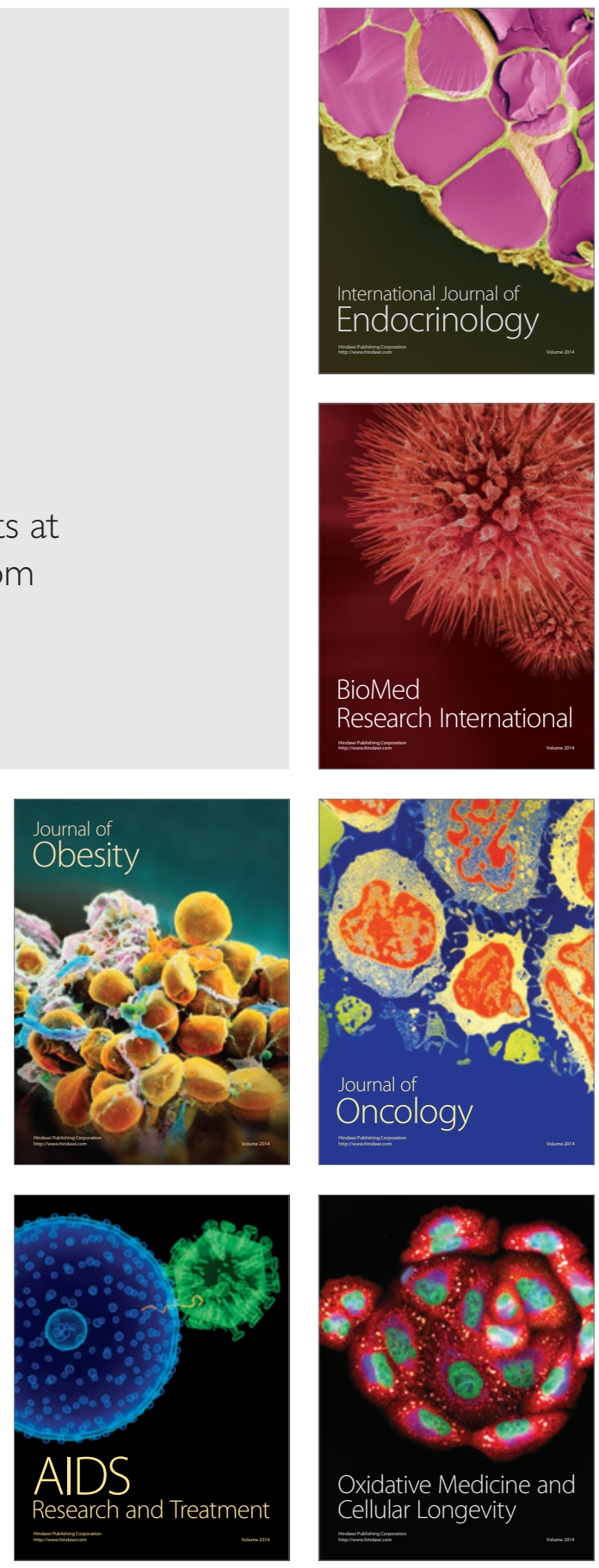